\section{Tuberculosis in Hemodialysis Patients in a Single Centre Tertiary Renal Care Unit}

Sir,

Tuberculosis (TB) is an infectious disease and an important cause of morbidity and mortality, especially in immunocompromised patients. Diagnosis of TB is crucial because it is spreadable by respiratory tract. Moreover, the disease is easily treatable and potentially preventable with prophylaxis. Many studies pointed out the increased rate of TB among patients with End-Stage Renal Disease (ESRD) in comparison with the general population. ${ }^{1-3}$ The reason behind the increased rate of TB among ESRD patients is related to the change in immune response, which is associated with uremic toxins and exacerbated by hemodialysis (HD). ${ }^{4}$ TB in HD patients has different clinical presentations than the general population; and difficult to diagnose with conventional techniques.

In this study, 71 (2.1\%) patients were found to have TB out of 3,000 patients on maintenance HD over a period of 17 years. Age range was 18-73 years. The female to male ratio for TB was 1:1.23. Diabetes was present in $25(35.2 \%)$ patients, while $31(43.7 \%)$ patients were hypertensive. Fever was the most common presenting complain in $56(79 \%)$ patients followed by cough in 43 $(60.6 \%)$ patients, while $13(18.3 \%)$ patients had night sweats. Pulmonary TB was present in $8(11.3 \%)$ patients while extra pulmonary TB was found in $79 \%$ of the cases. X-ray chest showed a pathological appearance in $53.5 \%$ of the cases, out of which pleural effusion was noted in $31(43.7 \%)$ cases. Abdominal TB was found in $17(23.9 \%)$ patients; and in these patients, ascitic fluid revealed high lymphocyte counts. Spinal TB was present in $4(5.6 \%)$ patients. TB culture was positive in 4 patients sent from pleural fluid and in one patient from ascitic fluid. In biopsy of 8 patients who had cervical lymphadenopathy, T.B was positive in $5(7 \%)$ patients.

Anti-tuberculous treatment (ATT) was started empirically in $5(7 \%)$ patients as these patients had fever of unknown origin without obvious abnormality on physical examination and other laboratory parameters. According to the therapeutic patterns, the patients received 4 drugs regime of ATT for $9.39 \pm 1.64$ months, in which 66 (93\%) recovered, while $5(7 \%)$ died during the course of disease.

Dialysis patients are immunocompromised due to various factors and are more prone to develop TB. In these patients, active disease is not obvious as in other non-immunocompromised patients and the prevalence of latent TB in patients is high; therefore, screening for latent TB in this population is suggested, aiming to prevent progression to active TB and secondary contamination to others.

\section{REFERENCES}

1. Malik GH, Al-Harbi AS, Al-Mohaya S, Al-Khawajah H, Kechrid M, Al-Hassan $\mathrm{AO}$, et al. Eleven years of experience with dialysis associated tuberculosis. Clin Nephrol 2002; 58: 356-62.

2. Malik GH, Al-Mohaya SA, Al-Harbi AS, Kechrid M, Azhari O, Shetia $S$, et al. Spectrum of tuber-culosis in dialysis patients in Saudi Arabia. Saudi J Kidney Dis Transpl 2003; 14:145-52.

3. Li SY, Chen TJ, Chung KW, Tsai LW, Yang WC, Chen JY, et al. Mycobacterium tuberculosis infection of end-satge renal disease patients in Taiwan: a nationwide longitudinal study. Clin Microbiol infect 2011; 17:1646-52.

4. Descamps-Latscha $B$. The immune system in end-stage renal disease. Curr Opin Nephrol Hypertens 1993; 2:883-91.

Ruqaya Qureshi and Salman Imtiaz

Department of Nephrology, The Kidney Centre Postgraduate Training Institute, Rafiqui Shaheed Road, Karachi, Pakistan

Correspondence: Dr. Ruqaya Qureshi, Department of Nephrology, The Kidney Centre Postgraduate Training Institute, 179/9, Rafiqui Shaheed Road, Karachi, Pakistan E-mail:dr_ruquayya@hotmail.com

Received: July 03, 2018; Accepted: September 14, 2018 